\begin{tabular}{|c|l|}
\hline Title & Thermoelectric A nalysis for Helical Power Generation Systems \\
\hline Author(s) & Meng, Xiangning; Fujisaka, Takey uki; Suzuki, Ryosuke O. \\
\hline Citation & $\begin{array}{l}\text { Journal of Electronic Materials, 43(6), 1509-1520 } \\
\text { https://doi.org/40.1007/311664 013-2768-8 }\end{array}$ \\
\hline Issue Date & 201406 \\
\hline Doc URL & http://hdl.handle.net/2115/59121 \\
\hline Rights & The final publication is available at link.springer.com \\
\hline Type & article (author version) \\
\hline File Information & revised manuscript.pdf \\
\hline
\end{tabular}

Instructions for use 


\title{
Thermoelectric analysis for helical power generation systems
}

\author{
Xiangning Meng ${ }^{12}$ Takeyuki Fujisaka $^{1}$ Ryosuke O. Suzuki $^{13}$
}

Contact e-mails: mengxn@eng.hokudai.ac.jp and rsuzuki@eng.hokudai.ac.jp

1. Faculty of Engineering, Hokkaido University, Sapporo, Hokkaido 060-8628, Japan

2. School of Materials and Metallurgy, Northeastern University, Shenyang 110819, China

3. Japan Science and Technology Agency, JST 


\section{Abstract}

Because the conversion efficiency of thermoelectric (TE) is still an issue, the performance of a three-dimensional helical thermoelectric generation (TEG) system is examined by exposing it to a temperature difference with hot and cold sources. The helical paths for the two thermal fluids give the TEG device the potential to efficiently convert thermal energy. The characteristic performances of the helical system are numerically analyzed by using the finite-volume method in a compact system. The helical system is compared with a straight system in which all the TE elements are established to have an equivalent geometry. The difference between the TE performances of the two systems is not significant when the TE surfaces are maintained at constant temperatures. Therefore, the helical system is a reasonable and feasible design because of its merits, such as compactness and larger and homogenous temperature difference. Both the electromotive force and the current in the TEG system increase linearly with a temperature difference $\Delta T$ applied at the two module surfaces. However, the TE performance is affected by the geometry of the helical system. The current preferentially flows through a mainstream path determined by the geometry of the TE element.

Key words: Thermoelectric generation; Helical system; Heat transfer; Finite element analysis 


\section{Introduction}

Heat can be directly converted into electricity in a thermoelectric (TE) generation (TEG) system, which is based on the Seebeck effect at a junction of two different materials. This method has some advantages, including the ability to use inexhaustible resources and low-grade energy, such as renewable solar heat and unrecovered waste-heat, because it is noiseless, pollution-free, and reliably operates in isolated places. Additionally, chemical reactions or mechanical moving parts are no longer needed. Many previous studies focused on the improvement of the inherent properties of thermoelectric materials ${ }^{[1-5]}$. However, the low conversion efficiency is still a distinct disadvantage ${ }^{[6-9]}$. Another factor in maximizing the efficiency is the optimal design of the TEG as well as the improvement of the TE materials ${ }^{[10]}$. Optimizing the TEG design is critical for obtaining the maximum performance because the output power from the TEG system can be significantly enhanced by combining the TE elements thermally and electrically in series ${ }^{[11]}$. For example, the number of $p-n$ pairs, the shape of the multi-stage TE panels, and the module geometry were optimized to obtain the best performance ${ }^{[12-17]}$.

The electromotive force of a TEG is the sum of the multiplication of the relative Seebeck coefficient $S$ and the temperature difference $\Delta T$ of the thermoelements for all serial connections. Therefore, the main issue in obtaining a larger output power is providing a lager $\Delta T$ for all the TE modules because $S$ is determined only by the materials used. A compact system for large-scale power generation was proposed that included a multi-panel consisting of TE modules, which efficiently provides a larger $\Delta T$ using multiple fluid paths. Suzuki et al. ${ }^{[18,19]}$ conducted a mathematic evaluation of the power generations with cylindrical multi-tubes, roll-cake TE tubes and TE double cylinders. The multi-panels can significantly shorten the TE device length, while only slightly decreasing the output. A unique design of a double helical exposed to two thermal fluids in a counter flow was also proposed by seamless three-dimensional connection ${ }^{[20]}$, as seen in Figure 1. Its design aims for a homogeneous $\Delta T$ for all TE panels by recovering the heat loss through a TE panel, supplying the heat to another 
fluid, and passing the captured heat to a different TE panel. The internal friction and pressure loss due to the contact with the path wall can be also minimized with a smooth fluid rotation. In a previous paper ${ }^{[20]}$, only a rough idea was proposed for this process, but no evaluation was carried out as to whether it was effective. The effect of the geometric parameters, such as the pitch and the diameter were not studied, and the heat exchange through the TE panel and the thermal flow of hot and cold fluids require more explored. A fluid dynamics simulation is crucial to simplify the design and the optimization by solving the necessary thermal and fluid conditions.

The purpose of this fundamental work was to illustrate the basic phenomenon of a helical TEG under a fixed temperature field, and to clarify the effects of the bent elements three-dimensionally by conducting a computational simulation of a physical model of one helical circuit. The contribution of the thermal fluids will be reported separately.

\section{Modeling}

\subsection{Finite-Element Entity Model}

The concept of the double helical TEG is visually demonstrated in Figure 1, where the TE panels are composed of p-type and n-type elements that are connected together in series, and the hot and cold fluids flow into and out of the channels, leading to a smooth stream of thermal fluids. A counter flow of two fluids can shorten the total height of a TE generator, which lowers the cost for practical construction. The pitch of a TE panel is important for evaluating the performance of this generator, which should be analyzed via a three-dimensional method. As an example, Figure 2 depicts a single-cycle of a TE panel, which consists of four circuits connected in series, and each circuit has the same number of p-n pairs. Because of complicated structure of multiple-geometric variables, an extensive research is needed to fully understand the effects of the variables. The size, shape, layout 
and links for each p-n junction will be discussed separately in detail. Here, one circuit in this TE panel is used as an example. A straight system is also designed in which all the elements are rectangular and connected in a straight line. The elements in the straight system have equivalent dimensions as those in the helical system. In order to compare the performance of helical and straight systems, both systems consist of the same number of TE pairs, and all the corresponding parts, such as p-type and n-type elements, electrodes and insulator, have the same volume. The only difference between the two systems is that all the parts are bent in the helical system, facilitating a more compact structure. An entity model for the helical system is three-dimensionally meshed, as illustrated in Figure 3 (a). This is used to conduct the finite-element simulations for the analysis of the TE process. The bent TE pairs in the helical system are illustrated in Figure 3 (b). The equivalent pairs in the straight system are shown in Figure 3 (c). The properties of the materials are listed in Table 1. For simplification, the following assumptions are made for formulating of the models: (1) the entire TE system is adiabatic to the outside; (2) perfect tight bonding exists among all parts; and (3) the properties of the materials are temperature independent.

\subsection{Analytical Model}

A model of the heat balance in the TE system is illustrated as shown in Figure 4. This balance is expressed as Equation (1) and Equation (2), when we consider from the macroscopic view. The theoretical output power is deduced as Equation (3).

$$
\begin{aligned}
& Q_{1}=Q_{\mathrm{c}}+Q_{\mathrm{P} 1}-0.5 Q_{\mathrm{J}} \\
& Q_{\mathrm{c}}+Q_{\mathrm{P} 2}+0.5 Q_{\mathrm{J}}=Q_{2}
\end{aligned}
$$




$$
P=Q_{\mathrm{P} 1}-Q_{\mathrm{P} 2}-Q_{\mathrm{J}}
$$

where $Q_{1}$ and $Q_{2}$ are the heat conducted through the layers of the insulator and the electrode, respectively, and $Q_{\mathrm{P} 1}$ and $Q_{\mathrm{P} 2}$ are the Peltier heat that, depend on the temperature and the current, respectively. These heats appear at the interfaces between the electrodes and the TE modules. $T_{\mathrm{h}}$ and $T_{\mathrm{c}}$ are temperature at these interfaces, and $T_{1}$ and $T_{2}$ are the temperature of the hot and cold surfaces, respectively. $T_{1}$ and $T_{2}$ are constant in this work. $Q_{\mathrm{J}}$ is the Joule heat following the Joule law, $Q_{\mathrm{c}}$ is the total heat conducted through all the TE modules, and $P$ is the output power of the TEG. The electric resistance and the output energy of the TEG are written as Equation (4) and Equation (5), respectively. The maximum output is obtained by Equation (6) at an optimal electric resistance. Here, an internal electric resistance is initially evaluated using Equation (4), but the optimal value is determined by repeated iterations, as mentioned later.

$$
\begin{aligned}
& R_{\mathrm{i}}=\sum_{\mathrm{k}=1}^{\mathrm{m}}\left(\rho_{\mathrm{p}}^{\mathrm{k}} \frac{d_{\mathrm{p}}^{\mathrm{k}}}{A_{\mathrm{p}}^{\mathrm{k}}}+\rho_{\mathrm{n}}^{\mathrm{k}} \frac{d_{\mathrm{n}}^{\mathrm{k}}}{A_{\mathrm{n}}^{\mathrm{k}}}\right) \\
& P=I^{2} R_{\mathrm{i}}=\frac{S^{2}\left(T_{\mathrm{h}}-T_{\mathrm{c}}\right)^{2}}{\left(R_{\mathrm{i}}+R_{\mathrm{e}}\right)^{2}} R_{\mathrm{i}} \\
& P_{\max }=\frac{S^{2}\left(T_{\mathrm{h}}-T_{\mathrm{c}}\right)^{2}}{4 \mathrm{R}_{\mathrm{o}}}
\end{aligned}
$$

where the subscripts $\mathrm{p}$ and $\mathrm{n}$ represent the p-type and n-type materials, $R_{\mathrm{i}}, R_{\mathrm{e}}$ and $R_{\mathrm{o}}$ are the internal, external and optimal electric resistances of the TE system, and $d$ and $A$ are the length and cross-sectional area of the TE modules, respectively. $S$ is the relative Seebeck coefficient, $\rho$ is the electric resistivity, $I$ is the electric current, 
and $m$ is the number of $p-n$ pairs.

\subsection{Constitution Equation}

The heat balance is unable to provide information on the distributions of the temperature and the current density inside the TE materials. Differential equations for the heat conduction for each finite-element volume are solved based on energy conservation. The heat conduction equation under steady-state conditions is written as Equation (7).

$$
\nabla \cdot(\lambda \Delta T)+\rho|\boldsymbol{J}|^{2}-T \boldsymbol{J} \cdot \nabla S=0
$$

where the three terms represent the heat conduction, the Joule heat generated by the current along the finite-element volume, and the heating or cooling generated by the Thomson effect, respectively. $\lambda$ is the thermal conductivity and $\boldsymbol{J}$ is the current density determined by the electric potential and the temperature using Equation (8).

$$
\rho J=-\nabla V-S \nabla T
$$

where the voltage drop in Ohm's law is expressed by the change of electric potential and by the voltage generated from the Seebeck effect. The differential Equation (9) is derived from Equation (8) by applying charge conservation under steady-state conditions.

$$
\nabla \cdot\left(-\frac{1}{\rho} \nabla V\right)=\nabla \cdot\left(-\frac{s}{\rho} \nabla V\right)
$$


The temperature and electric potential distributions are obtained by solving the simultaneous differential equations. Equation (7) and Equation (9) numerically based on the finite-volume method using the commercial software, FLUENT. The contribution of the TE phenomenon was originally coded in FLUENT and combined with FLUENT conventional codes ${ }^{[14]}$. The evaluation of the current density was originally codes in our project ${ }^{[21]}$ because the optional function delivered by ANSYS Co. Ltd. did not contain the calculation of current density. Because the final results gradually converge with each complex calculation for the entire TE process, the iteration was repeated approximately 5000 times or longer until a sufficient convergence was achieved.

\section{Results and Discussion}

\subsection{Performance Comparison}

Numerical simulations for helical and straight systems were conducted, in which the equivalent dimensions were established as shown in Figure $\mathbf{3}$ and the detailed configuration data are listed in Table 2. The thermo-physical properties of p-type and n-type materials were assumed to be constant in the temperature range of $300 \mathrm{~K}-600 \mathrm{~K}$. The temperature of the cold surface, $T_{2}$, was fixed at $300 \mathrm{~K}$ to represent an ambient temperature, and the temperature of the hot surface, $T_{1}$, is sequentially changed to $400 \mathrm{~K}, 450 \mathrm{~K}, 500 \mathrm{~K}, 550 \mathrm{~K}$ and $600 \mathrm{~K}$. Here, the optimal electric resistances of the internal circuit for the two systems were calculated by repeated iterations based on Equation (4) in the FLUENT environment.

The changes of the voltage and the current due to the temperature difference $\Delta T$, i.e., $\Delta T=T_{1}-T_{2}$, between the hot and the cold junctions of the TE elements in the two TE systems are depicted in Figure 5. Both the voltage and the current increased linearly with $\Delta T$, from $0.295 \mathrm{~V}$ and $1.253 \mathrm{~A}$ at a $\Delta T$ of $100 \mathrm{~K}$ to $0.876 \mathrm{~V}$ and $3.72 \mathrm{~A}$ at a $\Delta T$ of $300 \mathrm{~K}$ in the helical system, and from $0.297 \mathrm{~V}$ and $1.261 \mathrm{~A}$ at a $\Delta T$ of $100 \mathrm{~K}$ to $0.884 \mathrm{~V}$ and $3.753 \mathrm{~A}$ at a 
$\Delta T$ of $300 \mathrm{~K}$ in the straight system. The increments of the voltage and the current are $0.145 \mathrm{~V}$ and $0.62 \mathrm{~A}$, respectively, for a $\Delta T$ interval of $50 \mathrm{~K}$.

The output power of the TE system is obtained by multiplying the voltage and the current, and this value exhibits a parabolic rising trend because of the constant temperature at the module surfaces. The power increased from $0.37 \mathrm{~W}$ at $\Delta T=100 \mathrm{~K}$ to $3.26 \mathrm{~W}$ at $\Delta T=300 \mathrm{~K}$ in the helical system, and from $0.375 \mathrm{~W}$ to $3.319 \mathrm{~W}$ in the straight system, as shown in Figure 6 (a). Naturally, the temperature derivative of the output power of the helical system increases linearly, and the temperature derivative of the output power of the straight system is slightly higher than that of the helical system, as shown in Figure 6 (b).

Although the TE performance of helical systems is slightly depressed, no significant performance changes between the two systems are found based on the above-mentioned results. The precise differences of the TE performance is compared in Table 3, where $r_{\mathrm{vol}}, r_{\mathrm{cur}}$ and $r_{\text {pow }}$ are the differences of the voltage, current and output power, respectively, in the two systems as defined by Equation (10).

$$
r_{i}=\frac{P_{i}^{\mathrm{hel}}-P_{i}^{\mathrm{str}}}{P_{i}^{\mathrm{str}}}
$$

where $r$ is the difference ratio and $P$ is the TE performance. The subscript $i$ represents vol, cur and pow, which are the voltage, current and power, respectively. The superscripts hel and str represent the helical and straight systems, respectively.

The higher temperature results in a larger performance difference, although the differences of the voltage and the current between the two systems are less than $1 \%$, and the difference in the output power does not exceed $2 \%$. These evaluations were based on the assumption that the temperature is constant. The small drop of performance in the helical system is compensated by the merits of its design. If the heat of two thermal fluids in counter flow 
is exchanged to efficiently result in a larger and homogenous $\Delta T$ on the TE panels in a restricted space, a more compact system may be designed. Implementing this system would restrict the internal friction and the pressure loss of fluids simultaneously. These advantages are significant enough to counteract the small loss in TE performance compared to the equivalent straight system. The helical TE system is a reasonable and feasible design: it is sufficiently economical to neglect its slight decline in performance.

The TE performance at a $\Delta T$ of $200 \mathrm{~K}$, including the three-dimensional distributions of temperature, heat flux, voltage and current density is depicted in Figure $\mathbf{7}$ and Figure 8. These figures illustrate the TE phenomena occurring in the two TE systems under steady-state thermal conditions. The positive and negative heat fluxes represent the inflow and the outflow of heat in the TE system, in which the Peltier effect occurs, as well as heat conduction. The negative voltage represents the relative electromotive force because the interface between electrode and insulator was chosen as the origin. The series connection at the terminal reflects the difference in the electric potential.

All the three-dimensional distributions and the contour shape of the straight system are consistent, whereas the specific design of the helical system does not allow this. The isothermal surfaces in the straight system are parallel to the hot and cold surfaces of the TE panels with a fixed thermal boundary, as shown in Figure 7 (a). Conversely, those of the helical system are not strictly parallel to the hot and cold surfaces: the temperature gradient inside the TE modules was not uniform, as evidenced by the tilting of isothermal surfaces in Figure 8 (a). A common feature shared by the two systems is that the high heat flux tended to concentrate on the interfaces between the TE elements and the plate electrodes, the interfaces composed of n-type elements exhibited a larger flux than those composed of p-type elements. This behavior is because the heat accumulates from the lower thermal conductivity of n-type materials, as depicted in Figure 7 (b) and Figure 8 (b). The electromotive force is distributed throughout both systems from high potential to low potential, as shown in 
Figure 7 (c) and Figure 8 (c).

The current density is substantially impacted by the cross-sectional dimensions of the current flow. Hence, a higher current density occurs on the electrodes connected to the two TE elements, as shown in Figure 7 (d) and

Figure 8 (d). The distribution of current density in the straight system has a geometric symmetry because of the system shape, while diagonal inactive regions with low current density appeared at the bent TE elements in the helical system. Interestingly, these inactive regions are not always far from the high current density in the helical system. For example, the diagonal inactive regions marked by the red arrows in Figure 8 (d) exist close to the electrodes with a high current density, while the two pairs of diagonal inactive regions in the two adjacent TE elements are far from these high-density electrodes. This implies that a suitable path for the current exists, and the mainstream of the current is chosen spontaneously, reflecting the shape of the TE element. The current flow is designated by a red curve, as evidenced in Figure 8 (d). This mainstream path is governed by the detailed configuration and dimensions of the helical system, even by the connection patterns of the upstream and the downstream of the TE junctions. Therefore, a further in-depth study is required to assess an effective TEG design via rational optimization. Then, the performance of the TE system can be improved with an optimal system design, where excess parts can be removed to save fabrication materials and prevent heat loss.

\subsection{Features of Helical System}

\subsubsection{Voltage and Current Density}

The voltage and current density in the helical system are enlarged at a single pair of TE elements in Figure 9. A significantly high density appears at the central region of the electrode plate, which is a narrow channel for the current. The maximum current density in the TE elements arises at their corners that contact the central region of the electrode, as labeled by the black arrows in Figure 9 (a). This behavior is due to most of the electric charges 
carried in the electrode passing and flowing into the element at this point. Then the movement of these charges is weakened by the highly resistive elements. The retention of the current direction is strengthened by the shape mutation of the current path. The diagonal line connecting these two element corners with the maximum current density also indicates the position of the mainstream of the current in this element. The highest density in the plate electrodes appears at an intermediate position, where the inner arc on the side touches the TE elements. This position is subjected to a selection for the optimal flow path, as designated by the red arrows in Figure 9 (a). There is no symmetrical distribution of the current density in the entire TE junction because of the difference in electric resistivity of the p-type and n-type materials, which is clearly reflected by the distribution in the plate electrode. The electric charges move more readily through the p-type material with the lower electric resistivity. A higher amount of charge flows in the p-type elements than in the n-type elements. An apparent distribution inconsistency in the current density at the two terminals of the plate electrodes causes the charge discrepancy, which is indicated by two red rhomboids in Figure 9 (a). The lower and higher densities appeared at the terminals touching the p-type and n-type elements, respectively. The voltage at a TE junction in the electric circuit is presented in Figure 9 (c) and (d). An electric potential difference is generated in the conductors, as displayed by the color graduation, and the potential in the insulators is equal to zero. A TE junction in the helical system was radially separated into two parts at the geometric centerlines of the p-type and n-type elements and at the plate electrodes by the segments P-P, N-N, $\mathrm{E}_{\mathrm{h}}-\mathrm{E}_{\mathrm{h}}$ and $\mathrm{E}_{\mathrm{c}}-\mathrm{E}_{\mathrm{c}}$ as displayed in Figure 9 (b). The sectional distributions of current density inside these conductors were evaluated.

The current densities at the cross-sections along the lines of P-P and N-N are shown in Figure 10 (a) and Figure 10 (b), respectively. The density distributions presented on the two elements look similar. As mentioned previously, the higher density occurs in the vicinity of the interfaces between the elements and the electrodes. The maximum current density approaches $10 \mathrm{MA} \cdot \mathrm{m}^{-2}$ in the central region, while this high density gradually 
declines at the corners of the element side surfaces, dropping to approximately $1 \mathrm{MA} \cdot \mathrm{m}^{-2}$. The lower density is homogeneously distributed in the entire cross-sectional region. Interestingly, the region of lowest density appears between the regions of maximum density and homogeneous density, as shown by the grid areas in Figure 10 (a) and Figure 10 (b). The formation of these regions can be explained by the following: (1) the low-density regions are far away from the mainstream of the current, i.e., the high-density diagonal area between the two element corners with the maximum current density, and (2) a large amount of electric charge is collected in these two corners from the mainstream of the current, and only a small amount of charge is allocated to the other peripheral region. These regions confirmed the existence of the mainstream of the current in the TE system.

The current densities in the electrodes at the cross-sections along the lines of $E_{h}-E_{h}$ and $E_{c}-E_{c}$ are shown in Figure 11 (a) and Figure 11 (b), respectively. A current density as high as $27-28 \mathrm{MA} \cdot \mathrm{m}^{-2}$ homogenously distributes throughout the entire cross-section of a narrow channel with a thickness of $0.1 \mathrm{~mm}$. This distribution is significantly affected by the helical shape of the TE system and the density gradually decreased with a uniform gradient along a diagonal direction from the inner arc close to the TE element to the outer arc close to insulator, as indicated by the black arrows in Figure 11 (a) and Figure 11 (b). The density is distinctly reduced at the positions close to the surfaces contacting the hot and cold sources, while the higher density and homogenous gradient still exist at the corner regions on both sides of the electrode plates. This phenomenon is equivalent to that in the element corners in Figure (10). The formation of these local regions should be due to the accumulation of electric charges from insulator hindrance and insulated boundaries.

The maximum current densities of the above-mentioned cross-sections with different thermal conditions are shown in Figure 12. The maximum density linearly increased with the temperature difference $\Delta T$. The difference in electric resistivities led to the difference in current densities in p-type and n-type elements with the same dimensions. The maximum density of the n-type element is significantly higher than that of the p-type element at 
the same $\Delta T$. The maximum density is constant for the same $\Delta T$ for both electrodes touching the hot and cold surfaces because they have the same materials and dimensions. The increase in maximum densities are approximately evaluated at 3.8 MA $\cdot \mathrm{m}^{-2}, 5.3 \mathrm{MA} \cdot \mathrm{m}^{-2}$ and $7.0 \mathrm{MA} \cdot \mathrm{m}^{-2}$ for p-type elements, $\mathrm{n}$-type elements and electrodes for a $\Delta T$ interval of $50 \mathrm{~K}$, respectively.

\subsubsection{Temperature and Heat Flux}

The temperature and heat flux for a pair of p-n junctions in the helical system are depicted in Figure 13. Both values are homogenous in the conductors and the insulators because the thermal load was fixed at the boundary. The temperature distributions of the two elements are identical because the thermal conductivities of the two materials are nearly equivalent. The angle formed between the helical shape and the heat conduction direction makes the hierarchical isothermal surfaces close to the hot and cold sources to be not parallel to each other, while the isothermal surfaces in central positions are parallel because of the constant temperature gradient, as shown in Figure 13 (a). A slightly higher heat flux in the insulators appears at the positions corresponding to the junction with the lower thermal conductivity, as designated by the red rectangle in Figure 13 (b). This is due to the weak capacity of heat transfer of the n-type element, inevitably resulting in a high heat flow for a constant temperature and total heat.

The sectional distributions of temperature inside both types of elements and electrode plates are shown in Figure 14. The temperature distributions inside the elements are identical for the p-type and n-type materials, as depicted in Figure 14 (a). The temperature gradient is as high as $190 \mathrm{~K} \cdot \mathrm{mm}^{-1}$, which is higher than that of insulators, $10 \mathrm{~K} \cdot \mathrm{mm}^{-1}$. This gradient is caused by the large difference in the thermal conductivity of the materials. A narrow temperature range exists in the thin electrode plates, while a distinct gradient exists in the entire region of the cross-sections approximately $25 \mathrm{~K} \cdot \mathrm{mm}^{-1}$, and a small gradient exists in the region close to the surfaces of 
the hot and cold sources, less than $3 \mathrm{~K} \cdot \mathrm{mm}^{-1}$. In addition, a region resembling a quarter-circle shape with a relatively high temperature appeared in the position of the inner arc, as indicated by the black arrows in Figure 14 (b) and Figure 14 (c), respectively. These quarter-circle regions correspond to the area with the highest current density in the electrode plates and are caused by Joule heat.

\section{Conclusions}

(1) The TE performances of helical and straight TE systems were numerically evaluated in three-dimensional models. The voltage and current increased linearly with the temperature difference $\Delta T$ between the hot and cold sources in a TE system. The output power exhibited a parabolic increase with respect to $\Delta T$. No significant difference was found between the two systems with equivalent thermal conditions and dimensions. The differences in the voltage and the current between the two systems are less than $1 \%$, and the difference in the output power is within $2 \%$. These differences are totally inadequate to be compared with the sufficient economy caused by the merits of helical design. Therefore, the helical geometry has a satisfactory potential to be a TE generator.

(2) The TE performance is affected by the geometry of a helical system. A pair of diagonal inactive regions with a low current density was found, and the shortest path for the current was analyzed both in the elements and the electrodes. The maximum density arises at the element corners in contact with the central region of the electrodes. The maximum density in the electrode appears at the intermediate position of the inner arc on the side touching the TE elements. The current preferentially flows in a mainstream path. The maximum current density linearly increases with $\Delta T$. 


\section{Acknowledgements}

The authors are especially grateful to the technical supports by Dr. Min Chen in Denmark and to the financial supports of JST-CREST, Research Grant-in-Aid of JSPS (No. 2402077) and JSPS Fellowship (No. P12077). 


\section{References}

1. T. Aoki, C. Wan, H. Ishiguro, H. Morimitsu, K. Koumoto, J. Ceram. Soc. Jpn. 119, 382 (2011).

2. Y. Saiga, K. Suekuni, S.K. Deng, T. Yamamoto, Y. Kono, N. Ohya, T. Takabatake, J. Alloy. Compd. 507, 1 (2010).

3. T. Sakamoto, T. Iida, A. Matsumoto, Y. Honda, T. Nemoto, J. Sato, T. Nakajima, H. Taguchi, Y. Takanashi, J. Electron. Mater. 39, 1708 (2010).

4. A.J. Zhou, T.J. Zhu, X.B. Zhao, S.H. Yang, T. Dasgupta, C. Stiewe, R. Hassdorf, E. Mueller, J. Electron. Mater. 39, 2002 (2010).

5. Y. Ma, Q. Hao, B. Poudel, Y. Lan, B. Yu, D. Wang, G. Chen, Z. Ren, Nano Lett. 8, 2580 (2008).

6. K. Koumoto, R. Funahashi, E. Guilmeau, Y. Miyazaki, A. Weidenkaff, Y. Wang, C. Wan, J. Am. Ceram. Soc. 96, 1 (2013).

7. G. Min, M. Rowe, IEEE Trans. Energy Convers. 22, 528 (2007).

8. J. Zhu, J. Gao, M. Chen, J. Zhang, Q. Du, L.A. Rosendahl, R.O. Suzuki, J. Electron. Mater. 40, 744 (2011).

9. R.O. Suzuki, Y. Sasaki, T. Fujisaka, M. Chen, J. Electron. Mater. 41, 1766 (2012).

10. S.B. Riffat, G.Q. Qiu, Int. J. Energy Res. 30, 67 (2006).

11. R.O. Suzuki, J. Power Sources 133, 277 (2003).

12. A.Z. Sahin, B.S. Yilbas, Energy Conv. Manag. 65, 26 (2013).

13. A. Takezawa, M. Kitamura, Int. J. Numer. Methods Eng. 90, 1363 (2012).

14. M. Chen, L.A. Rosendahl, T. Condra, Int. J. Heat Mass Transf. 54, 345 (2011).

15. R. Funahashi, Sci. Adv. Mater. 3, 682 (2011).

16. L. Zhang, T. Tosho, N. Okinaka, T. Akiyama, Mater. Trans. 49, 1675 (2008).

17. M. Chen, S.J. Andreasen, L. Rosendahl, S.K. Kaer, and T. Condra, J. Electron. Mater. 39, 1593 (2010). 
18. R.O. Suzuki, D. Tanaka, J. Power Sources 124, 293 (2003).

19. R.O. Suzuki, D. Tanaka, J. Power Sources 132, 266 (2004).

20. R.O. Suzuki, D. Tanaka, J. Power Sources 122, 201 (2003).

21. T. Fujisaka, H. Sui, R.O. Suzuki, J. Electron. Mater. (2013) doi: 10.1007/s11664-012-2400-3. 


\section{Figure captions}

Figure 1 Schematic representation of a double helical TEG.

Figure 2 Single-cycle TE panel in helical TEG system.

Figure 3 Meshed entity models of a helical system for finite-element analysis.

Figure 4 Schematic representation of heat balance of TE system.

Figure 5 Effect of $\Delta T$ on voltage (a) and current (b).

Figure 6 Effect of $\Delta T$ on output power (a) and its increment (b).

Figure 7 TE phenomena occurring in straight system.

Figure 8 TE phenomena occurring in helical system.

Figure 9 Current density and voltage in helical system.

Figure 10 Current density $\left(\mathrm{A} \cdot \mathrm{m}^{-2}\right)$ inside p-type (a) and n-type (b) elements.

Figure 11 Current density $\left(\mathrm{A} \cdot \mathrm{m}^{-2}\right)$ inside electrodes of contacting with hot (a) and cold (b) insulators.

Figure 12 Effect of $\Delta T$ on maximum current density of elements and electrodes.

Figure 13 Temperature (a) and heat flux (b) in helical system.

Figure 14 Temperature inside TE elements (a) and electrodes (b) and (c). 
Table captions

Table 1 Thermal and electric properties of materials.

Table 2 Configuration data of two systems.

Table 3 Difference of TE performance between two systems. 


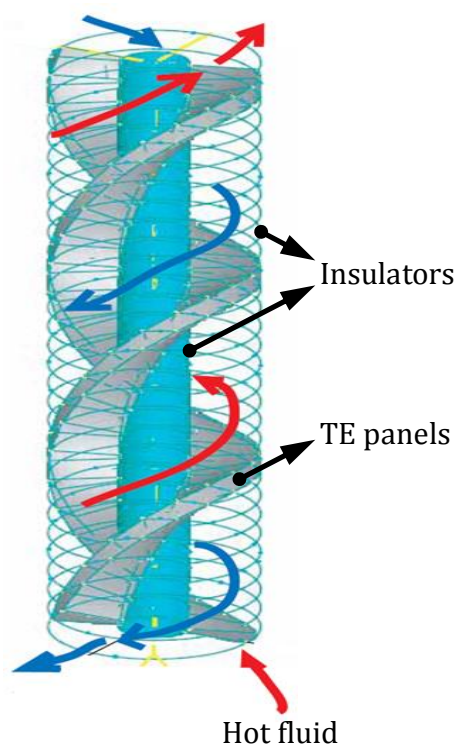

Figure 1 Schematic representation of a double helical TEG

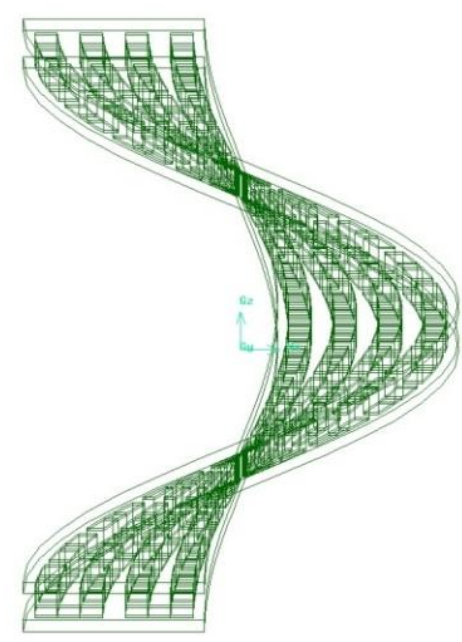

Figure 2 Single-cycle TE panel of helical TEG system 


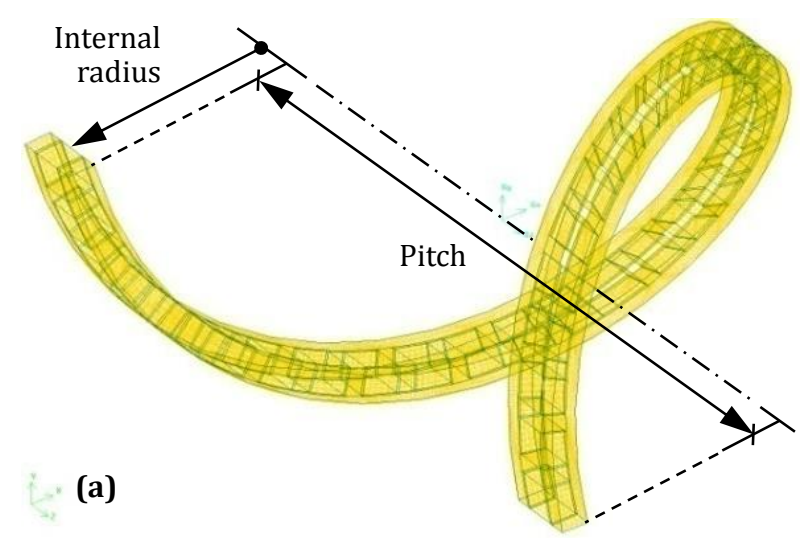

(b)

(c)

Figure 3 Meshed entity models of helical systems for finite-element analysis

Table 1 Thermal and electric properties of materials

\begin{tabular}{rllccc}
\hline & & \multicolumn{2}{c}{ Figure-of-Merit } & Thermal conductivity & Electric resistivity \\
& & - & $\mathrm{W} \cdot \mathrm{m}^{-1} \cdot \mathrm{K}^{-1}$ & $10^{-6} \Omega \cdot \mathrm{m}$ \\
\hline $\mathrm{Bi}_{2} \mathrm{Te}_{3}$ & (p-type) & $>0.9$ & $(300-600 \mathrm{~K})$ & 2.06 & 5.5 \\
$\mathrm{Bi}_{2} \mathrm{Te}_{3}$ & (n-type) & $>0.9$ & $(300-600 \mathrm{~K})$ & 2.02 & 10.0 \\
$\mathrm{Cu}$ & (electrode) & & 398 & 0.0155 \\
$\mathrm{Al}_{2} \mathrm{O}_{3}$ & (insulator) & & 36 & \\
\hline
\end{tabular}

Constant temperature / Hot surface

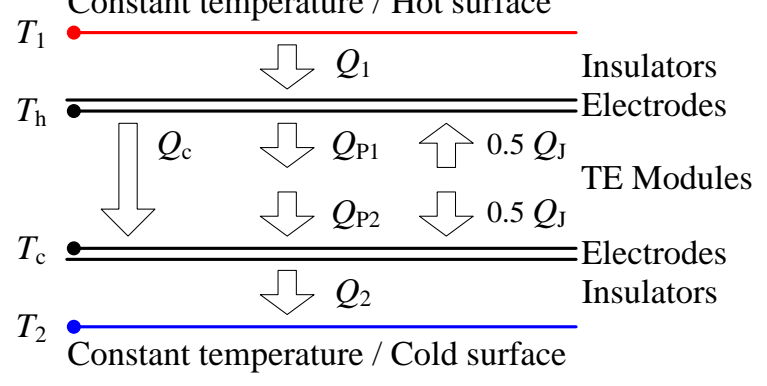

Figure 4 Schematic representation of heat balance of TE system 
Table 2 Configuration data of two systems

\begin{tabular}{|c|c|c|c|c|c|c|c|}
\hline \multirow{3}{*}{ System } & \multirow{3}{*}{ p-n pairs } & \multicolumn{4}{|c|}{ Elements (p-type / n-type) } & \multirow{3}{*}{$\begin{array}{c}\text { Electrode } \\
\text { Thickness } \\
\text { mm }\end{array}$} & \multirow{3}{*}{$\begin{array}{c}\text { Insulator } \\
\text { Thickness } \\
\text { mm }\end{array}$} \\
\hline & & Area & Leg length & Radii (outer / inner) & Width & & \\
\hline & & $\mathrm{mm}^{2}$ & $\mathrm{~mm}$ & $\mathrm{~mm}$ & $\mathrm{~mm}$ & & \\
\hline Helical & 18 & 1.0 & 1.0 & $9.6 / 8.6$ & - & 0.1 & 0.5 \\
\hline Straight & 18 & 1.0 & 1.0 & - & 1.0 & 0.1 & 0.5 \\
\hline
\end{tabular}
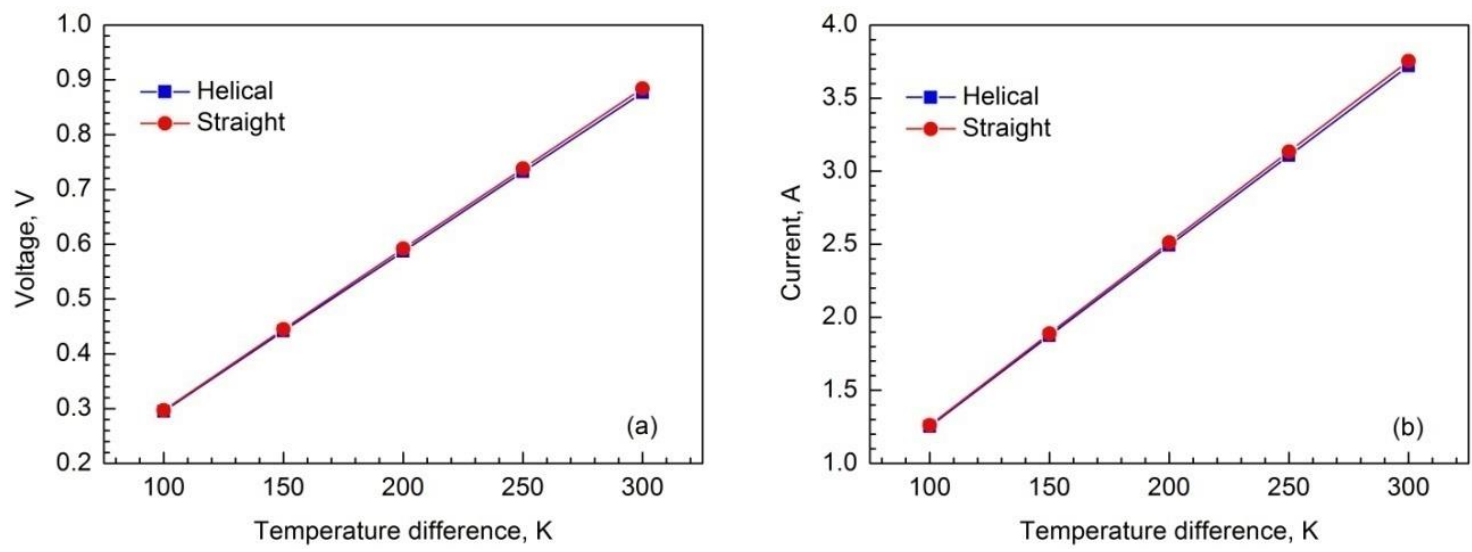

Figure 5 Effect of $\Delta T$ on voltage (a) and current (b)


Figure 6 Effect of $\Delta T$ on output power (a) and its increment (b) 
Table 3 Difference of TE performance between two systems

\begin{tabular}{cccc}
\hline$\Delta T$ & $r_{\text {vol }}$ & $r_{\text {cur }}$ & $r_{\text {out }}$ \\
$\mathrm{K}$ & $\%$ & $\%$ & $\%$ \\
\hline 100 & -0.70 & -0.70 & -1.40 \\
150 & -0.75 & -0.75 & -1.49 \\
200 & -0.80 & -0.80 & -1.59 \\
250 & -0.84 & -0.84 & -1.68 \\
300 & -0.89 & -0.89 & -1.77 \\
\hline
\end{tabular}
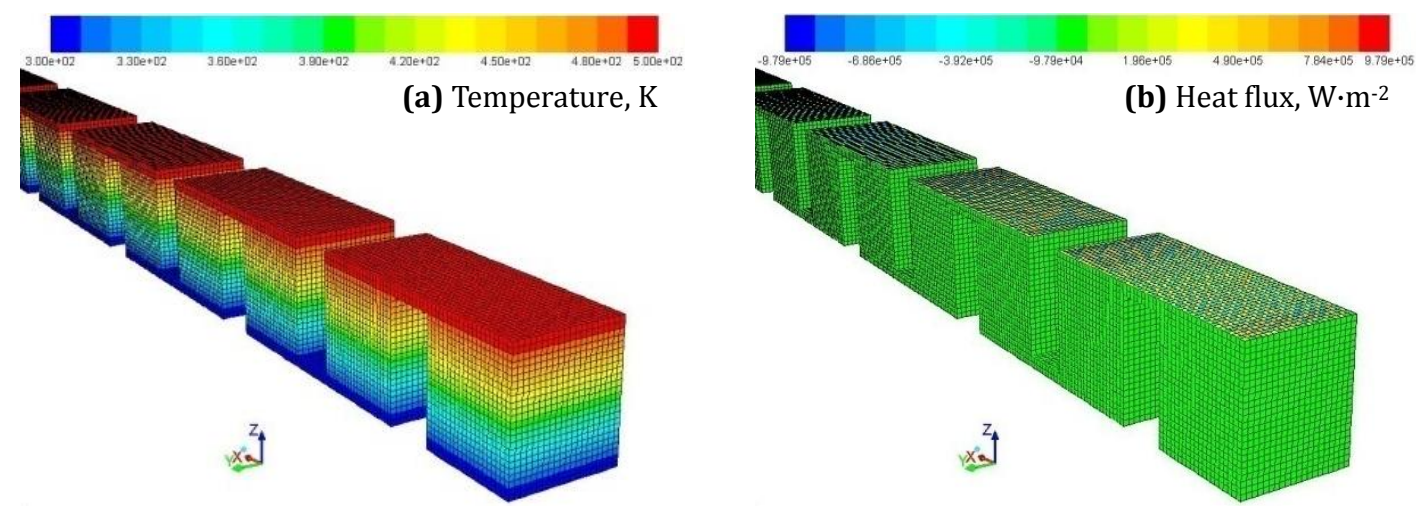

(b) Heat flux, $\mathrm{W} \cdot \mathrm{m}^{-2}$

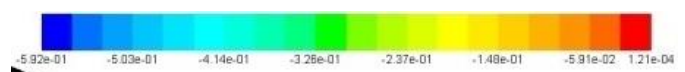

(c) Voltage, V
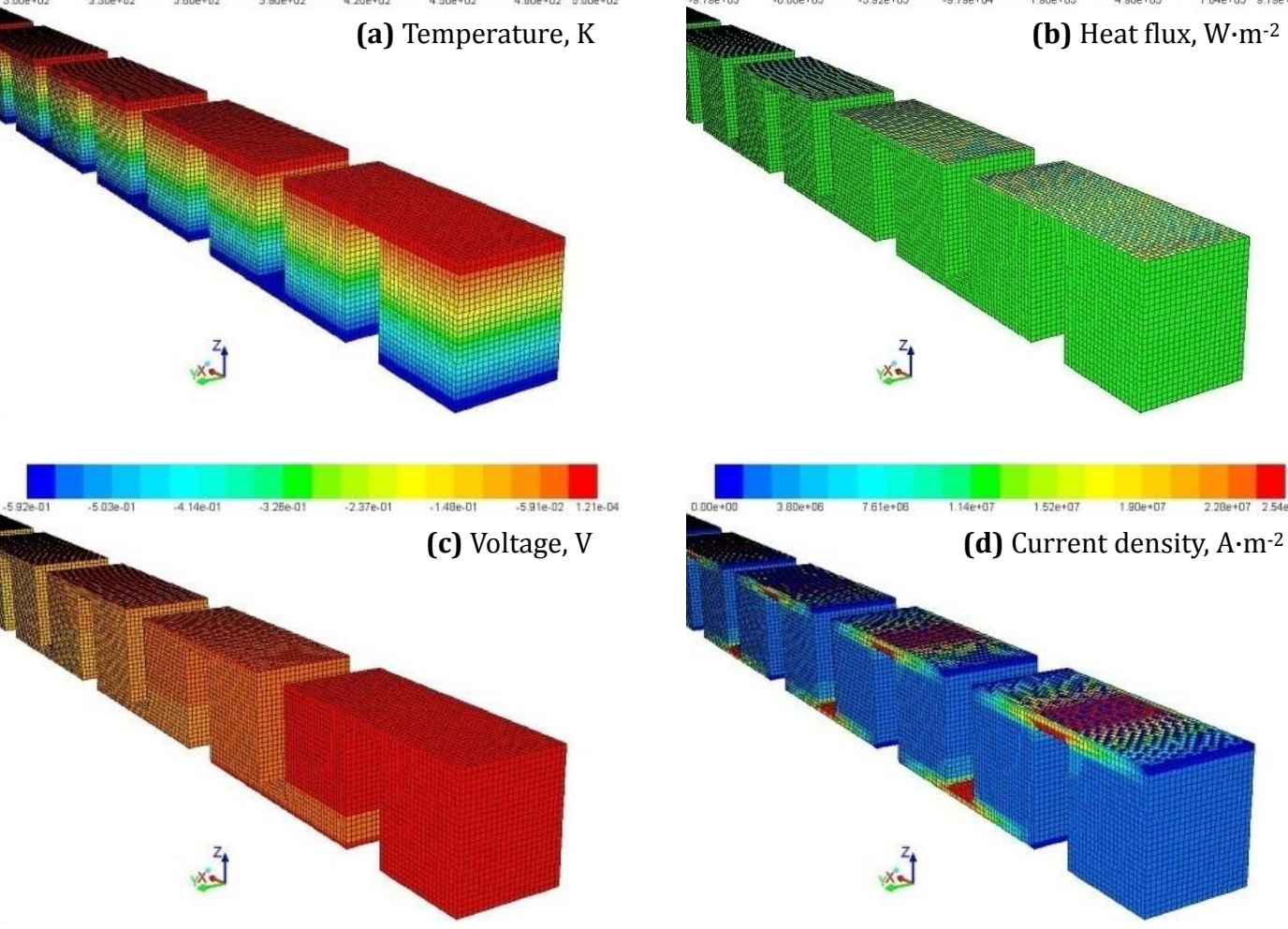

Figure 7 TE phenomena occurring in straight system 

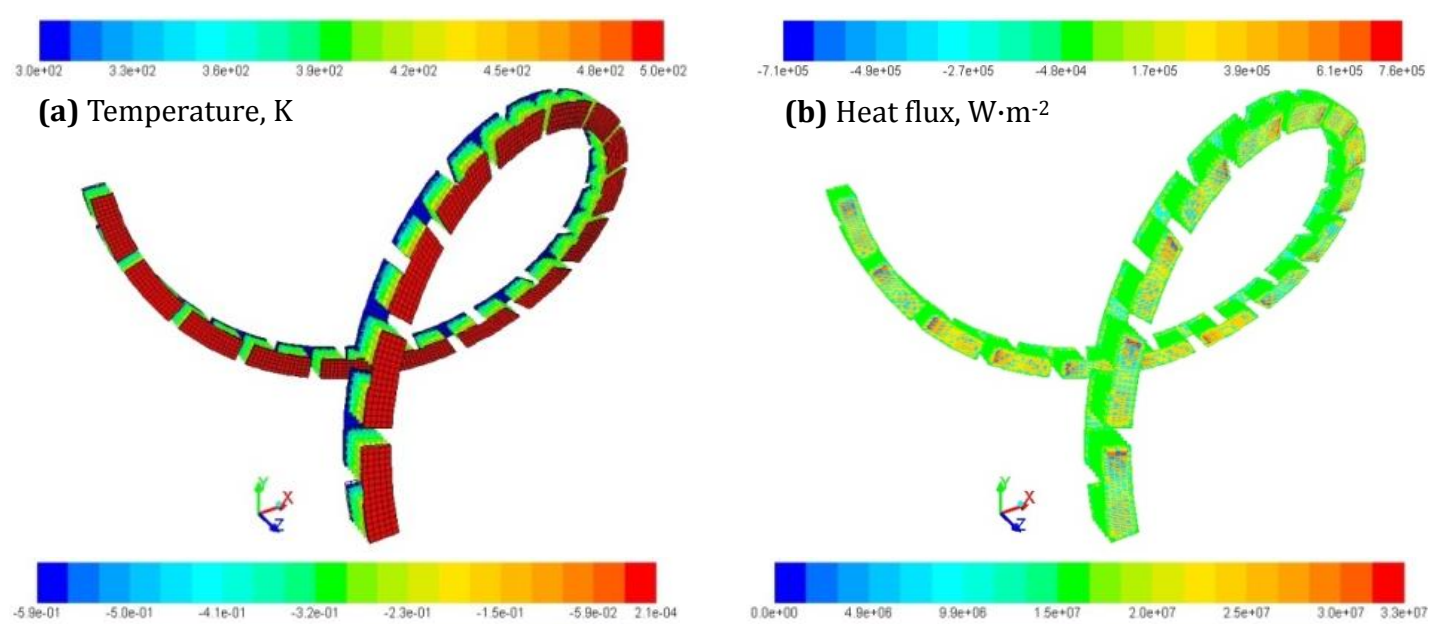

(b) Heat flux, $\mathrm{W} \cdot \mathrm{m}^{-2}$

(c) Voltage, V
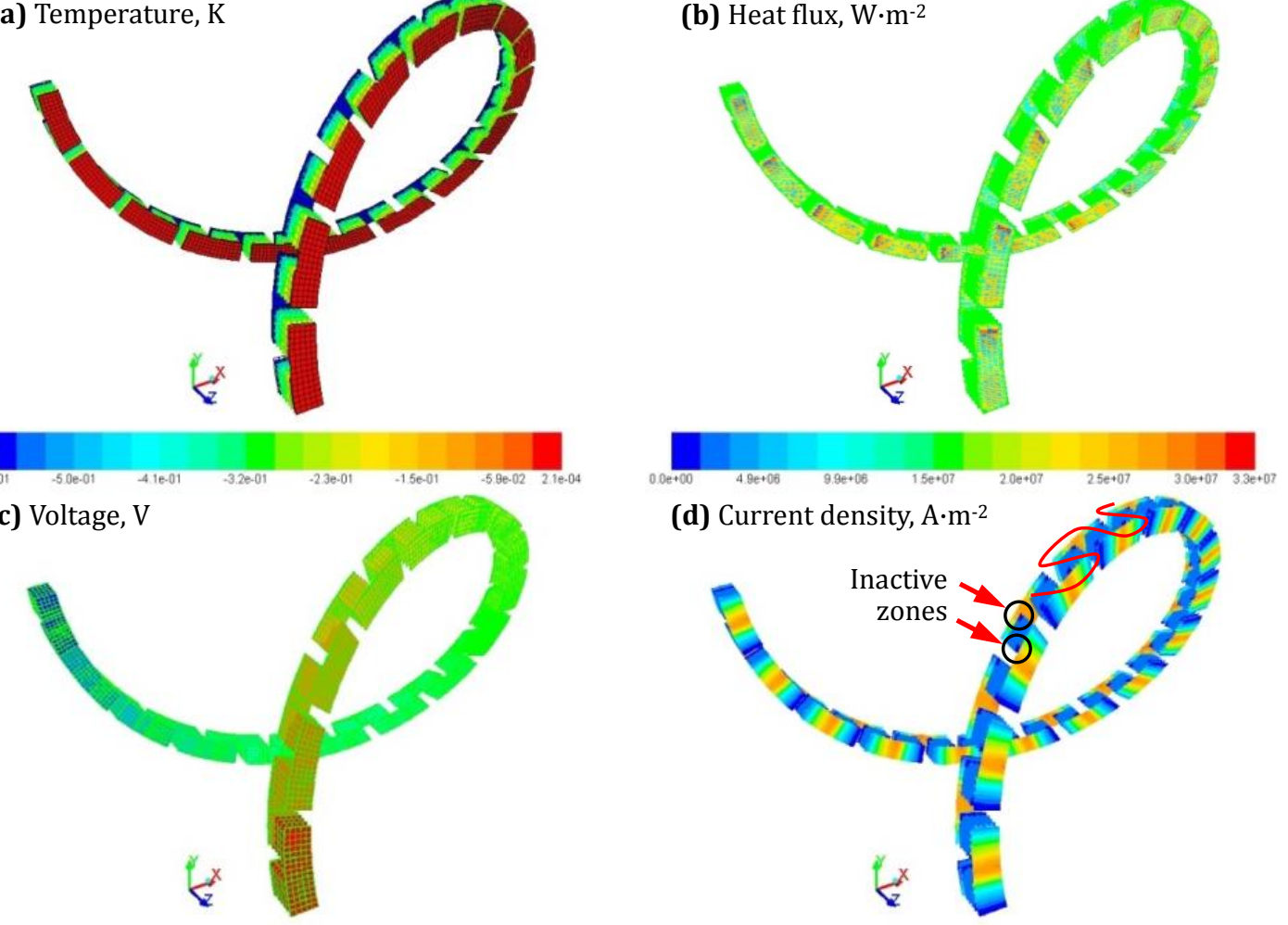

(d) Current density, $\mathrm{A} \cdot \mathrm{m}^{-2}$

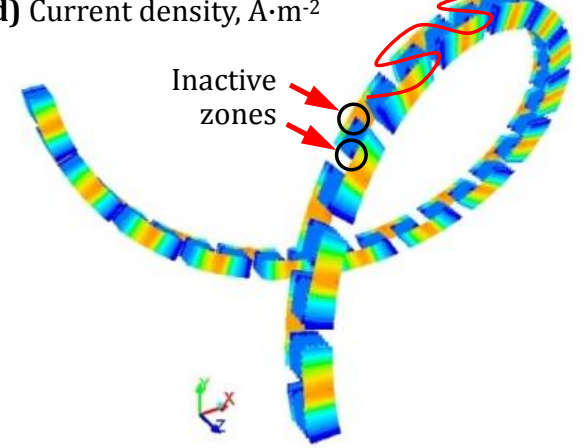

Figure 8 TE phenomena occurring in helical system 

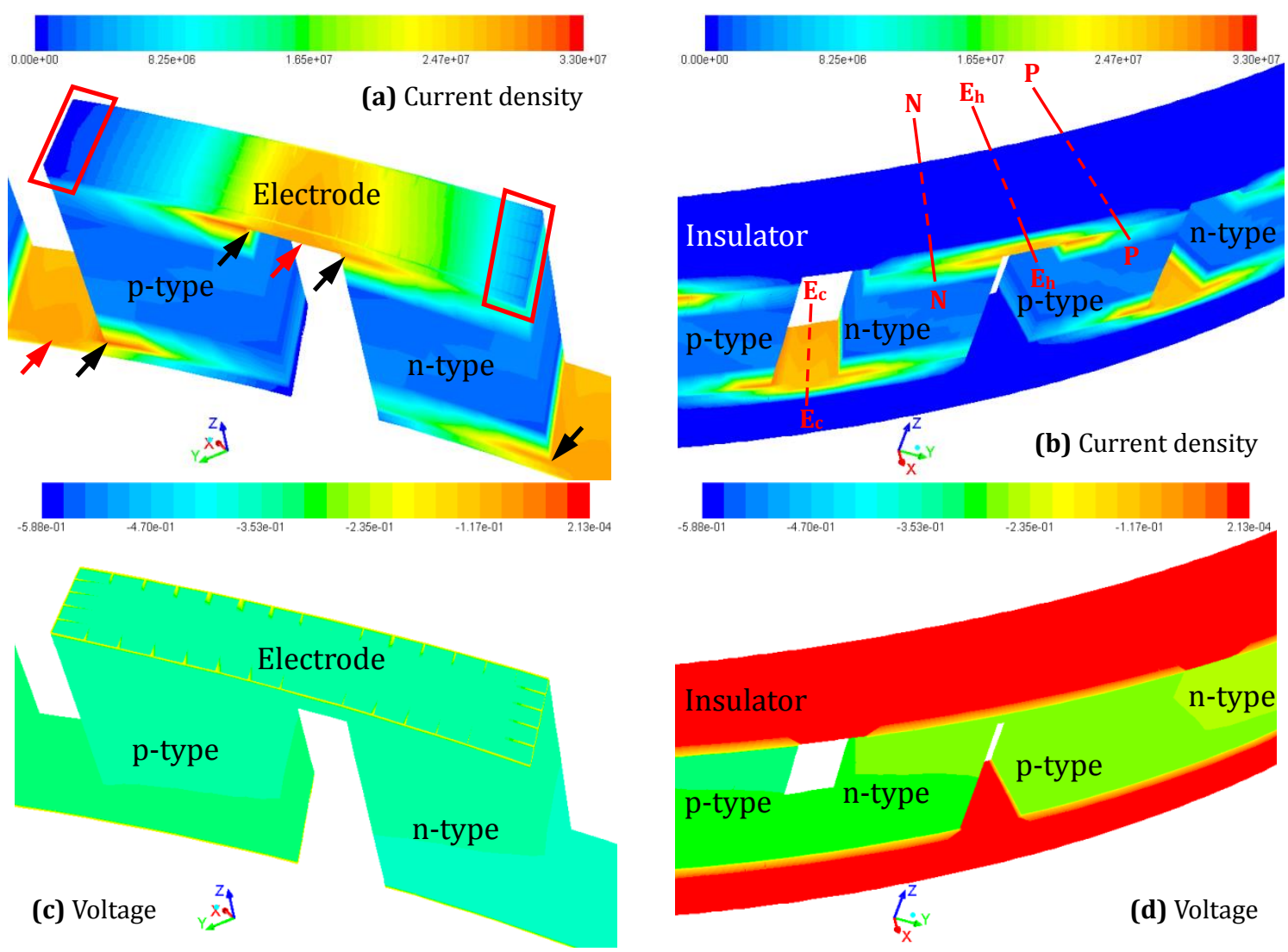

Figure 9 Current density $\left(\mathrm{A} \cdot \mathrm{m}^{-2}\right)$ and voltage $(\mathrm{V})$ in helical system
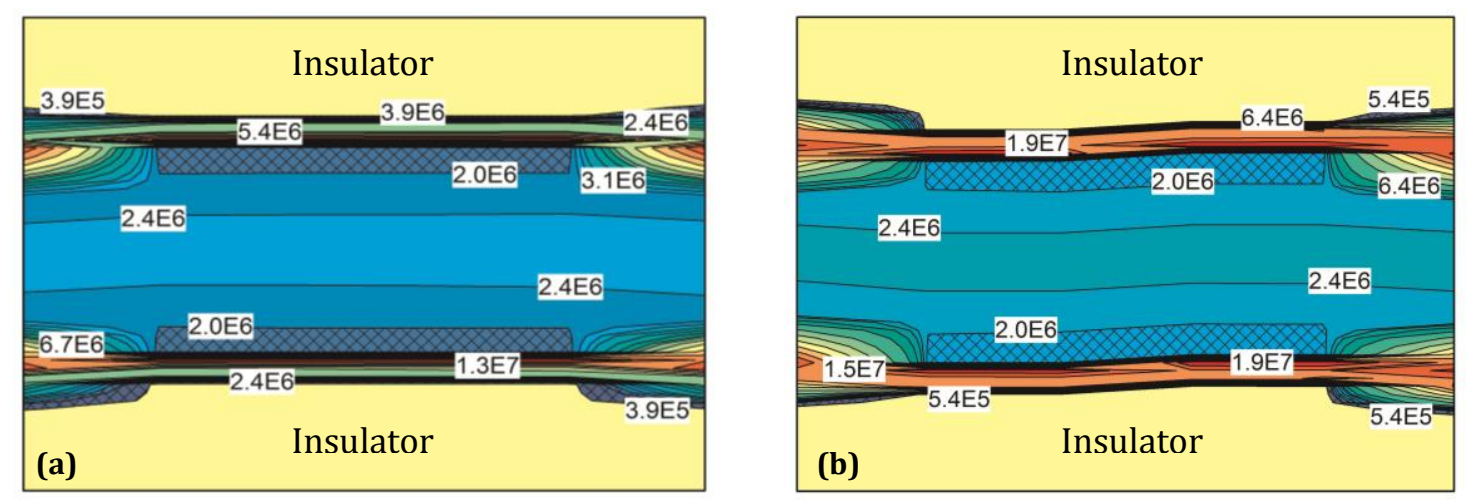

Figure 10 Current density $\left(A \cdot m^{-2}\right)$ inside p-type (a) and n-type (b) elements 

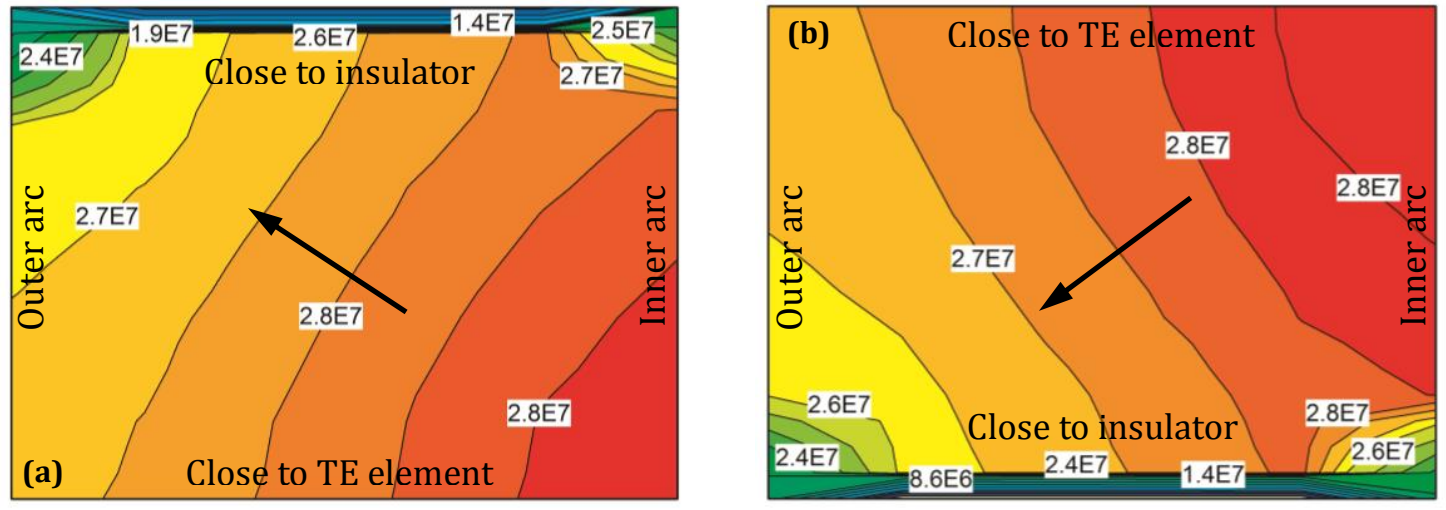

Figure 11 Current density $\left(A \cdot \mathrm{m}^{-2}\right)$ inside electrodes of contacting with hot (a) and cold (b) insulators

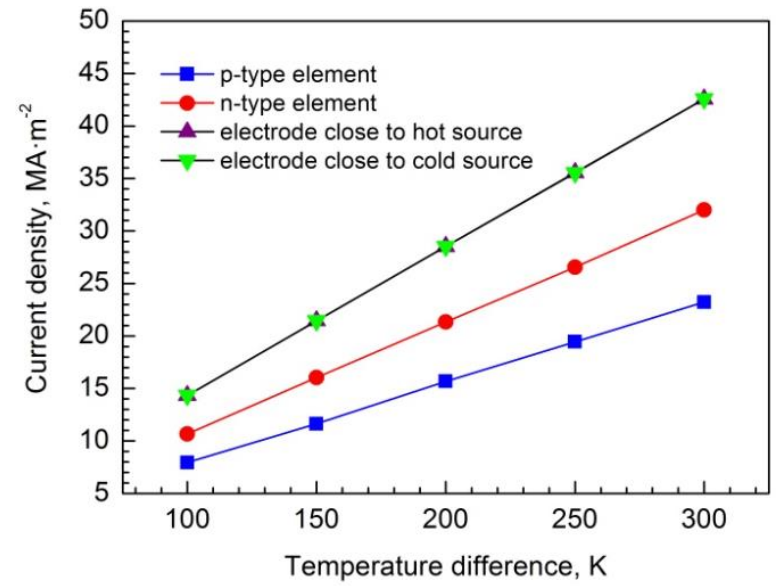

Figure 12 Effect of $\Delta T$ on maximum current density of elements and electrodes 

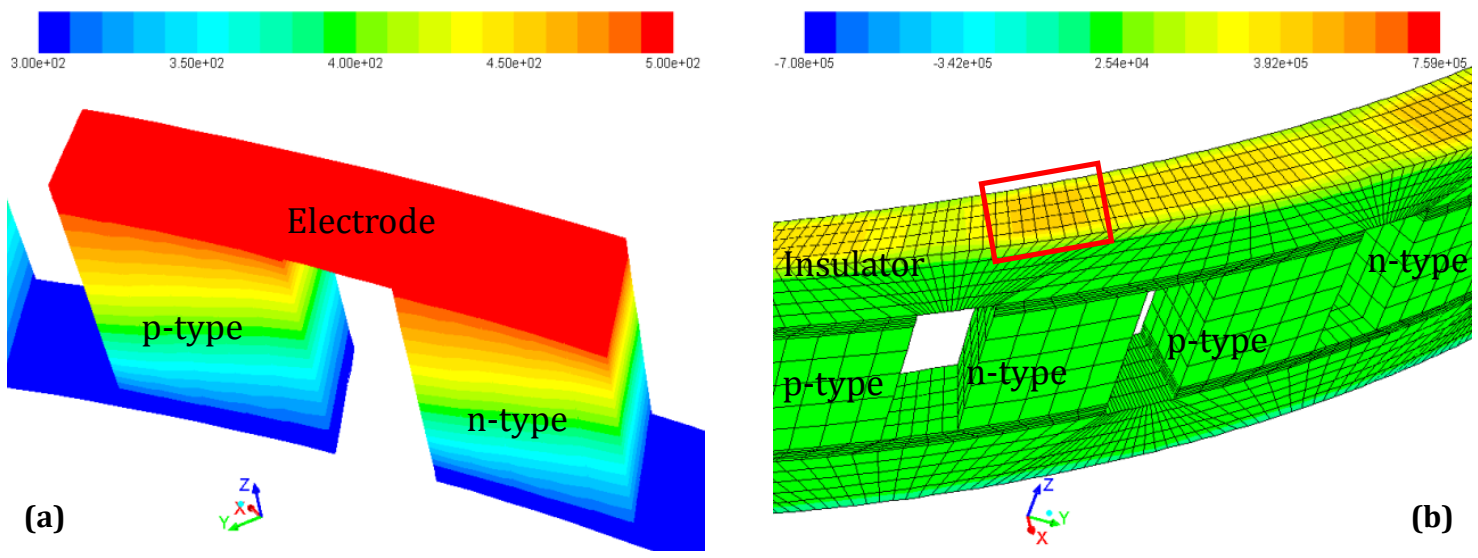

Figure 13 Temperature (K) (a) and heat flux $\left(\mathrm{W} \cdot \mathrm{m}^{-2}\right)(\mathrm{b})$ in helical system
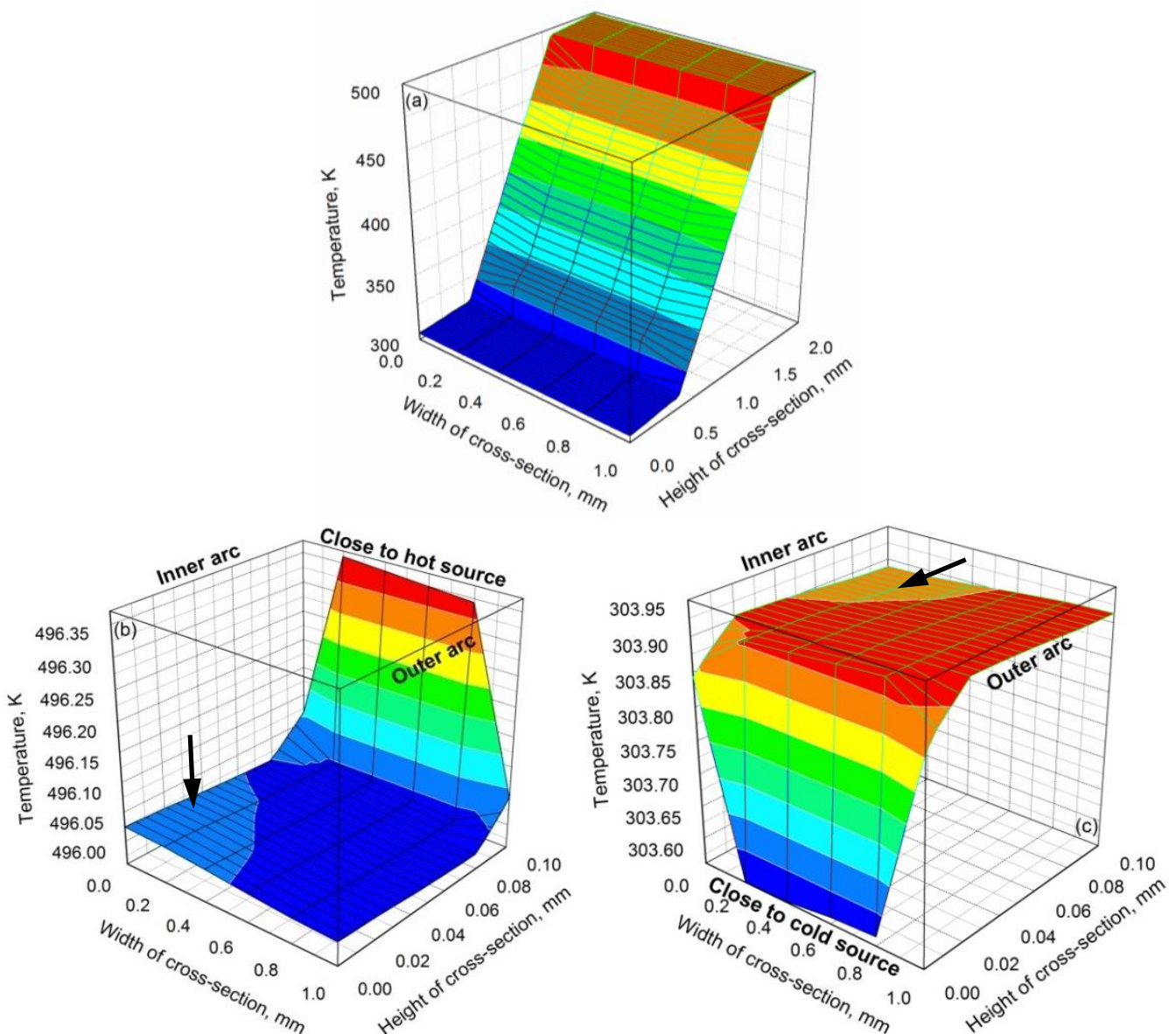

Figure 14 Temperature (K) inside TE elements (a) and electrodes (b) and (c) 